\title{
Global Adaptive Output Feedback Tracking Control of an Unmanned Aerial Vehicle
}

\author{
W. MacKunis, Z. D. Wilcox, M. K. Kaiser, and W. E. Dixon
}

\begin{abstract}
An output feedback (OFB) dynamic inversion control strategy is developed for an unmanned aerial vehicle (UAV) that achieves global asymptotic tracking of a reference model. The UAV is modeled as an uncertain linear time-invariant (LTI) system with an additive bounded nonvanishing nonlinear disturbance. A continuous tracking controller is designed to mitigate the nonlinear disturbance and inversion error, and an adaptive law is utilized to compensate for the parametric uncertainty. Global asymptotic tracking of the measurable output states is proven via a Lyapunovlike stability analysis, and high-fidelity simulation results are provided to illustrate the applicability and performance of the developed control law.
\end{abstract}

Index Terms-Adaptive control, dynamic inversion (DI), Lyapunov methods, nonlinear control, robust control.

\section{INTRODUCTION}

D YNAMIC INVERSION (DI) is a similar concept as feedback linearization that is commonly used within the aerospace community to replace linear aircraft dynamics with a reference model. Parametric uncertainty and unmodeled disturbances present in the dynamic model can cause complications in DI-based control design due to the resulting DI error. While DI-based control techniques have been successfully applied to systems containing parametric uncertainty in the corresponding dynamic models (e.g., see [1]-[4]), input-multiplicative uncertainty merits special attention. One method to address uncertainty in the input matrix is to use a robust control approach based on high gain or high frequency feedback. For example, a sliding-mode controller is designed in [5] for an agile missile model containing aerodynamic uncertainty. The scalar input uncertainty in [5] was bounded and damped out through a discontinuous sliding-mode control element. A discontinuous sliding mode controller was also developed in [6] for attitude tracking of an unpowered flying vehicle with an uncertain column deficient non-symmetric input matrix. While discontinuous sliding-mode controllers (SMC) are capable of compensating for inversion error, the instantaneous switching exhibited by such controllers (i.e., the "chattering" phenomenon) is undesirable for practical aircraft with rate limited

Manuscript received September 09, 2009; revised November 06, 2009. Manuscript received in final form November 10, 2009. First published December 22, 2009; current version published October 22, 2010. Recommended by Associate Editor N. Hovakimyan. This work was supported in part by the NSF CAREER Award 0547448, by the NSF Award 0901491, and by the Department of Energy under Grant DE-FG04-86NE37967 as part of the DOE University Research Program in Robotics (URPR)

W. MacKunis, Z. D. Wilcox, and W. E. Dixon are with the Mechanical and Aerospace Engineering Department, University of Florida, Gainesville, FL 32611-6250 (e-mail: mackunis@gmail.com; zibrus@ufl.edu; wdixon@ufl.edu).

M. K. Kaiser is with the Mosaic Atm, Inc., Leesburg, VA 20175 USA (e-mail: kkaiser@mosaicatm.com).

Digital Object Identifier 10.1109/TCST.2009.2036835 actuators. Motivated by the need to eliminate infinite switching to compensate for disturbances, a continuous (i.e., finite rate) DI control design is developed in this brief, which is capable of achieving asymptotic reference model tracking for a linear time-invariant (LTI) aircraft model with linear in the parameters (LP) uncertainty in the nonsquare (column deficient) input matrix and additive bounded nonlinear disturbances. This result builds on our preliminary work in [7], where a continuous robust controller achieves semi-global asymptotic tracking for an uncertain aircraft model with a column deficient input matrix; however, the controller in [7] requires the output measurements and the respective time derivatives.

As an alternative to robust control designs such as SMC, adaptive dynamic inversion (ADI) controllers seek to accommodate for model uncertainty without exploiting high gain or high frequency feedback. In [8], a full-state feedback adaptive control design was presented for a general class of fully-actuated nonlinear systems containing state-varying input uncertainty and a nonlinear disturbance that is linear in the uncertainty. The ADI design in [8] utilizes a matrix decomposition technique [9], [10] to yield a global asymptotic tracking result when the input uncertainty is assumed to be square and positive definite. A semi-global multiple-input-multiple-output (MIMO) extension is also provided in [8] using a robust controller for the case when the input matrix uncertainty is square, positive definite, and symmetric. A full-state feedback adaptive controller is developed in [11], which compensates for parametric uncertainty in a linearly parameterizable nonlinearity and a square input gain matrix. The approach in [11] applies a matrix decomposition technique to avoid singularities in the control law. An adaptive tracking controller is developed in [12] for nonlinear robot systems with kinematic, dynamic, and actuator uncertainties where the input uncertainty is a constant diagonal matrix. In our previous work in [13], an ADI controller is developed to achieve semi-global asymptotic tracking of an aircraft reference model where the aircraft dynamics contain column deficient nonsymmetric input uncertainty. However, like our robust DI controller in [7] the ADI controller in [13] also depends on the output states and the respective time derivatives.

Calculation of output derivatives can amplify the effects of noise and hinder controller performance. Motivated by the desire to reduce the effects of noise in the control system, output feedback control techniques have been widely investigated. The aforementioned full-state feedback adaptive technique in [11] is extended to an adaptive output feedback controller in [14] via the use of state estimators. In [15] and [16], adaptive output feedback controllers are designed for pitch and plunge motion control of an aeroelastic wing system. A backstepping-based design technique using state estimators is presented in [15], which 
requires measurement of pitch angle and plunge displacement. In [16], by obtaining a lower triangular form of the aeroelastic system via state transformation, flutter suppression is achieved using only pitch angle feedback. The control design in [15] is extended to compensate for unstructured uncertainty in [17]. The design in [17] uses an inverse control law along with a high gain observer to compensate for an unknown nonlinearly parameterizable function present in the dynamics. Two modular adaptive control systems are developed in [18], which use an estimation-based design to control pitch and plunge displacement of an aeroelastic wing. It is assumed in [18] that the sign of a control input coefficient is known along with the lower bound of its absolute value. An output feedback controller is presented in [19], which achieves flutter suppression and limit cycle oscillations in a nonlinear 2-D wing-flap system. Parametric uncertainty in the dynamic model is addressed using a Lyapunov-based adaptive law, and the unmeasurable states are compensated using state estimators. The controller in [19] is shown to regulate the pitch angle to a constant set point based on the assumption that the structures of the aeroelastic model and pitch spring nonlinearity are known. While the aforementioned ADI results compensate for parameteric uncertainty, they cannot be used to yield asymptotic tracking in the presence of unmodeled additive disturbances. The output feedback ADI missle pitch controller in [20] is an exception that can be applied to achieve robustness with respect to model uncertainties and disturbances, but the controller is based on a discontinuous SMC design. Neural network (NN)-based ADI controllers have been developed for aircraft with unstructured uncertainties in results such as (e.g., see [4], [21]-[26]). However, these results yield approximate tracking in the sense that they are limited to uniformly ultimately bounded tracking unless coupled with a discontinuous SMC element (e.g., [26]).

The contribution in this brief is the development of a continuous adaptive output feedback controller that achieves global asymptotic tracking of the outputs of a reference model, where the plant model contains a nonsquare, column deficient, uncertain input matrix and a nonvanishing bounded disturbance. In comparison with the results in [4]-[8], [11], [14]-[26], the developed controller utilizes a continuous robust feedback structure to compensate for the additive nonlinear disturbance along with an adaptive feedforward structure to compensate for parametric uncertainty. The current development exploits the matrix decomposition technique in [9], [10] so that the controller depends only on the output states, and not the respective time derivatives. Specifically, minimal knowledge of the UAV dynamic model is exploited along with the matrix decomposition technique to rewrite the tracking error dynamics in a form that is amenable to controller design. This manipulation enables design of a continuous adaptive output feedback control law that is capable of compensating for parametric input uncertainty and a nonvanishing additive disturbance. Global asymptotic tracking is proven via a Lyapunov-based stability analysis. To illustrate the practical performance of the proposed control design under realistic conditions, high fidelity numerical simulation results are provided, which take practical measurement noise and aircraft actuator position and rate constraints into account.

\section{SySTEM Model}

The subsequent development is based on the following UAV model [27]:

$$
\begin{aligned}
& \dot{x}=A x+B u+f(x, t) \\
& y=C x .
\end{aligned}
$$

In (1) and (2), $A \in \mathbb{R}^{n \times n}$ denotes a state matrix composed of unknown constant elements, $B \in \mathbb{R}^{n \times m}$ denotes a column deficient input matrix composed of uncertain constant elements with $m<n, C \in \mathbb{R}^{m \times n}$ denotes a known output matrix, $x(t) \in$ $\mathbb{R}^{n}$ denotes the state vector, $u(t) \in \mathbb{R}^{m}$ denotes a vector of control inputs, ${ }^{1}$ and $f(x, t) \in \mathbb{R}^{n}$ represents a state- and timedependent unknown, nonlinear disturbance. Based on (1) and (2), a reference model is defined as

$$
\begin{aligned}
\dot{x}_{m} & =A_{m} x_{m}+B_{m} \delta \\
y_{m} & =C x_{m}
\end{aligned}
$$

where $A_{m} \in \mathbb{R}^{n \times n}$ is Hurwitz, $B_{m} \in \mathbb{R}^{n \times m}$ is the reference input matrix, $\delta(t) \in \mathbb{R}^{m}$ is the reference input, $x_{m}(t) \in \mathbb{R}^{n}$ represents the reference states, $y_{m}(t) \in \mathbb{R}^{m}$ are the reference outputs, and $C$ is introduced in (2).

Property 1: The reference trajectory $x_{m}(t)$ is designed such that $x_{m}(t), \dot{x}_{m}(t), \ddot{x}_{m}(t), \dddot{x}_{m}(t) \in \mathcal{L}_{\infty} \forall t$.

Assumption 1: The nonlinear disturbance $f(x, t)$ and its first two time derivatives are assumed to exist and be bounded by known constants.

Assumption 1: A large magnitude disturbance (e.g., wind gust) could cause the aircraft to become unstable or uncontrollable; however, the subsequent development is based on the assumption that the dynamics in (1) are controllable.

For a discussion of nonlinearities that can be represented by $f(x, t)$ for an aircraft, see [7].

\section{Control DevelopMent}

\section{A. Control Objective}

The control objective is to ensure that the outputs $y(t)$ track the time-varying outputs generated from the reference model in (3) and (4). To quantify the control objective, an output tracking error, denoted by $e(t)=\left[e_{1}(t), e_{2}(t), \ldots, e_{m}(t)\right]^{T} \in \mathbb{R}^{m}$, is defined as

$$
e \triangleq y-y_{m}=C\left(x-x_{m}\right) .
$$

To facilitate the subsequent analysis, a filtered tracking error [28], denoted by $r(t)=\left[r_{1}(t), r_{2}(t), \ldots, r_{m}(t)\right]^{T} \in \mathbb{R}^{m}$, is defined as

$$
r \triangleq \dot{e}+\alpha e
$$

where $\alpha \in \mathbb{R}$ is a positive, constant control gain. The subsequent development is based on the assumption that only the

\footnotetext{
${ }^{1}$ The subsequent development assumes that $u(t) \in \mathbb{R}^{m}$ and $C \in \mathbb{R}^{m \times n}$ (i.e., the number of inputs is equal to the number of outputs). However, this control design can be applied to systems for which $u(t) \in \mathbb{R}^{p}$ and $C \in \mathbb{R}^{m \times n}$, where $p>m$ via the use of a pseudoinverse (e.g., Moore-Penrose) in the control law.
} 
output measurements $y(t)$ [and therefore $e(t)$ in (5)] are available. Hence, $r(t)$ is not measurable and is not used in the control development. The filtered tracking error is only introduced to facilitate the subsequent stability analysis.

To facilitate the subsequent robust output feedback control development and stability analysis, the state vector $x(t)$ will be segregated into measurable and unmeasurable components. This step will enable the segregation of terms that can be bounded as functions of the error states from those that are bounded by constants. To this end, the state vector $x(t)$ can be expressed as

$$
x(t)=\underline{x}(t)+x_{u}(t)
$$

where $\underline{x}(t) \in \mathbb{R}^{n}$ contains the $m$ output states, and $x_{u} \in \mathbb{R}^{n}$ contains the $n-m$ remaining states. Likewise, the reference states $x_{m}(t)$ can also be separated as in (7).

Assumption 3: The states $x_{u}(t)$ in (7) and the corresponding time derivatives can be further separated as

$$
x_{u}(t)=x_{\rho u}(t)+x_{\zeta u}(t) \quad \dot{x}_{u}(t)=\dot{x}_{\rho u}(t)+\dot{x}_{\zeta u}(t)
$$

where $x_{\rho u}(t), \dot{x}_{\rho u}(t), x_{\zeta u}(t), \dot{x}_{\zeta u}(t) \in \mathbb{R}^{n}$ are assumed to be upper bounded as

$$
\begin{aligned}
\left\|x_{\rho u}(t)\right\| \leq c_{1}\|z\| & \left\|x_{\zeta u}(t)\right\| \leq \zeta_{x u} \\
\left\|\dot{x}_{\rho u}(t)\right\| \leq c_{2}\|z\| & \left\|\dot{x}_{\zeta u}(t)\right\| \leq \zeta_{\dot{x} u}
\end{aligned}
$$

where $z(t) \in \mathbb{R}^{2 m}$ is defined as

$$
z \triangleq\left[\begin{array}{ll}
e^{T} & r^{T}
\end{array}\right]^{T}
$$

and $c_{1}, c_{2}, \zeta_{x u}, \zeta_{\dot{x} u} \in \mathbb{R}$ are known nonnegative bounding constants (i.e., the constants could be zero for different classes of systems).

\section{B. Open-Loop Error System}

The open-loop tracking error dynamics can be developed by taking the time derivative of (6) and utilizing the expressions in (1)-(4) to obtain

$$
\dot{r}=\tilde{N}_{0}+N_{d 0}+C A\left(\underline{\dot{x}}_{m}+\alpha \underline{x}_{m}\right)+C B(\dot{u}+\alpha u)
$$

where $\underline{x}_{m}(t) \in \mathbb{R}^{n}$ contains the reference states that correspond to the output states in $\underline{x}(t)$, and $\underline{\dot{x}}_{m}(t)$ denotes the respective time derivative. The auxiliary functions $\tilde{N}_{0}\left(x, \dot{x}, x_{m}, \dot{x}_{m}, t\right) \in$ $\mathbb{R}^{m}$ and $N_{d 0}\left(x, x_{m}, \dot{x}_{m}, \delta, \dot{\delta}, t\right) \in \mathbb{R}^{m}$ in (10) are defined as

$$
\tilde{N}_{0} \triangleq C A\left(\underline{\dot{x}}-\underline{\dot{x}}_{m}\right)+\alpha C A\left(\underline{x}-\underline{x}_{m}\right)+C A\left(\dot{x}_{\rho u}+\alpha x_{\rho u}\right)
$$

and

$$
-C A_{m}\left(\dot{x}_{m}+\alpha x_{m}\right)-C B_{m}(\dot{\delta}+\alpha \delta)
$$

Motivation for the selective grouping of the terms in (11) and (12) is derived from the fact that the following inequalities can be developed [29], [30]:

$$
\left\|\tilde{N}_{0}\right\| \leq \rho_{0}\|z\| \quad\left\|N_{d 0}\right\| \leq \zeta_{N_{d 0}} \quad\left\|\dot{N}_{d 0}\right\| \leq \zeta_{\dot{N}_{d 0}}
$$

where $\rho_{0}, \zeta_{N_{d 0}}, \zeta_{\dot{N}_{d 0}} \in \mathbb{R}$ are known positive bounding constants.

\section{Closed-Loop Error System}

Based on the expression in (10) and the subsequent stability analysis, the control input is designed as

$$
u=(C \hat{B})^{-1}\left(\mu_{0}-\mu_{1}\right)-\int_{0}^{t} \alpha u(\tau) d \tau
$$

where $\mu_{0}(t), \mu_{1}(t) \in \mathbb{R}^{m}$ denote subsequently defined feedback control terms, and $\hat{B} \in \mathbb{R}^{n \times m}$ is a constant feedforward estimate of the uncertain matrix $B$. After substituting the time derivative of (14) into (10), the error dynamics can be expressed as

$$
\dot{r}=\tilde{N}_{0}+N_{d 0}+C A\left(\underline{\dot{x}}_{m}+\alpha \underline{x}_{m}\right)+C B(C \hat{B})^{-1}\left(\dot{\mu}_{0}-\dot{\mu}_{1}\right) .
$$

Assumption 4: Upper and lower bounds of the uncertain input matrix $B$ are known such that the constant feedforward estimate $\hat{B}$ can be selected such that $C B(C \hat{B})^{-1}$ can be decomposed as follows [8]-[10], [31]:

$$
C B(C \hat{B})^{-1}=S T
$$

where $S \in \mathbb{R}^{m \times m}$ is symmetric and positive definite, and $T \in$ $\mathbb{R}^{m \times m}$ is a unity upper triangular matrix, which is diagonally dominant in the sense that

$$
\varepsilon \leq\left|T_{i i}\right|-\sum_{k=i+1}^{m}\left|T_{i k}\right| \leq Q \quad \forall i=1, \ldots, m-1 .
$$

In (17), $\varepsilon \in(0,1)$ and $Q \in \mathbb{R}^{+}$are known bounding constants, and $T_{i k} \in \mathbb{R}$ denotes the $(i, k)$ th element of the matrix $T$. Preliminary results indicate that this assumption is mild in the sense that the decomposition in (16) results in a diagonally dominant $T$ for a wide range of $\hat{B} \neq B$.

Based on (16), the error dynamics in (15) are

$$
S^{-1} \dot{r}=\tilde{N}_{1}+N_{d 1}+S^{-1} C A\left(\underline{\dot{x}}_{m}+\alpha \underline{x}_{m}\right)+T\left(\dot{\mu}_{0}-\dot{\mu}_{1}\right)-e
$$

where

$$
\tilde{N}_{1} \triangleq S^{-1} \tilde{N}_{0}+e \quad N_{d 1} \triangleq S^{-1} N_{d 0}
$$

Since $S$ is positive definite, the following inequalities can be developed:

$$
\left\|\tilde{N}_{1}\right\| \leq \rho_{1}\|z\| \quad\left\|N_{d 1}\right\| \leq \zeta_{N_{d 1}} \quad\left\|\dot{N}_{d 1}\right\| \leq \zeta_{\dot{N}_{d 1}}
$$


where $\rho_{1}, \zeta_{N_{d 1}}, \zeta_{\dot{N}_{d 1}} \in \mathbb{R}$ are positive bounding constants. The error dynamics in (18) can now be rewritten as

$$
S^{-1} \dot{r}=\tilde{N}_{1}+N_{d 1}+Y_{A} \theta_{A}+\bar{T} \dot{\mu}_{0}+\dot{\mu}_{0}-T \dot{\mu}_{1}-e
$$

where $\bar{T} \triangleq T-I_{m \times m}$ is a strictly upper triangular matrix, $I_{m \times m}$ is an $m \times m$ identity matrix, $Y_{A}\left(x_{m}, \dot{x}_{m}\right) \in \mathbb{R}^{m \times p_{1}}$ denotes a measurable regression matrix, and $\theta_{A} \in \mathbb{R}^{p_{1}}$ is a vector containing the unknown elements of the $A$ and $S$ matrices, defined via the parametrization

$$
Y_{A} \theta_{A} \triangleq S^{-1} C A\left(\underline{\dot{x}}_{m}+\alpha \underline{x}_{m}\right) .
$$

Based on the open-loop error dynamics in (20), the auxiliary control term $\mu_{0}(t)$ is designed as

$$
\begin{aligned}
\mu_{0} \triangleq & -\left(k_{s}+I_{m \times m}\right) e(t)+\left(k_{s}+I_{m \times m}\right) e(0) \\
& -\int_{0}^{t}\left[Y_{A} \hat{\theta}_{A}+\alpha\left(k_{s}+I_{m \times m}\right) e(\tau)\right] d \tau
\end{aligned}
$$

and the auxiliary control term $\mu_{1}(t)$ is designed as

$$
\mu_{1}=\int_{0}^{t} \beta \operatorname{sgn}(e(\tau)) d \tau
$$

where $\beta \in \mathbb{R}$ is a constant, positive control gain, $k_{s} \in \mathbb{R}^{m \times m}$ is a constant, positive definite, diagonal control gain matrix, and $\alpha$ is introduced in (6). The adaptive estimate $\hat{\theta}_{A}(t) \in \mathbb{R}^{p_{1}}$ in (22) is generated according to the adaptive update law

$$
\dot{\hat{\theta}}_{A i}=\operatorname{proj}\left(\Xi_{i}\right)
$$

where $\dot{\hat{\theta}}_{A i}(t)$ denotes the $i$ th component of $\dot{\hat{\theta}}_{A}(t)$, and $\Xi_{i}\left(x_{m}, \dot{x}_{m}, r\right)$ denotes the $i$ th component of $\Xi\left(x_{m}, \dot{x}_{m}, r\right) \forall i=1, \ldots, p_{1}$, where the auxiliary term $\Xi\left(x_{m}, \dot{x}_{m}, r\right) \in \mathbb{R}^{p_{1}}$ is defined as ${ }^{2}$

$$
\Xi\left(x_{m}, \dot{x}_{m}, r\right) \triangleq \Gamma_{A} Y_{A}^{T}\left(x_{m}, \dot{x}_{m}\right) r .
$$

For the adaptation law in (24) and (25), $\Gamma_{A} \in \mathbb{R}^{p_{1} \times p_{1}}$ is a constant, positive definite, symmetric adaptation gain matrix.

Property 2: The function $\operatorname{proj}(\cdot)$ in (24) denotes a normal projection algorithm, which ensures that the following inequality is satisfied (for further details, see [32]-[35]):

$$
\underline{\theta}_{A i} \leq \hat{\theta}_{A i} \leq \bar{\theta}_{A i}
$$

where $\underline{\theta}_{A i}, \bar{\theta}_{A i} \in \mathbb{R}$ denote known, constant lower and upper bounds, respectively, of $\hat{\theta}_{A i}(t) \forall i=1, \ldots, p_{1}$.

After substituting the time derivative of (22) into (20), the closed-loop error system can be determined as

$$
S^{-1} \dot{r}=\tilde{N}_{1}+\bar{T} \dot{\mu}_{0}+N_{d 1}-\left(k_{s}+I_{m \times m}\right) r+Y_{A} \tilde{\theta}_{A}-T \dot{\mu}_{1}-e
$$

where $\tilde{\theta}_{A}(t) \in \mathbb{R}^{p_{1}}$ denotes the parameter estimation error defined as

$$
\tilde{\theta}_{A} \triangleq \theta_{A}-\hat{\theta}_{A} .
$$

${ }^{2}$ Since the measurable regression matrix $Y_{A}(\cdot)$ contains only the reference trajectories $x_{m}$ and $\dot{x}_{m}$, the expression in (24) can be integrated by parts to prove that the adaptive estimate $\hat{\theta}_{A}(t)$ can be generated using only measurements of $e(t)$ (i.e., no $r(t)$ measurements, and hence, no $\dot{x}(t)$ measurements are required).
Using the time derivative of (22), the vector $\bar{T} \dot{\mu}_{0}$ can be expressed as

$$
\bar{T} \dot{\mu}_{0}=\left[\begin{array}{c}
\sum_{j=2}^{m} \bar{T}_{1 j} \dot{\mu}_{0 j} \\
\sum_{j=3}^{m} \bar{T}_{2 j} \dot{\mu}_{0 j} \\
\vdots \\
\bar{T}_{(m-1) m} \dot{\mu}_{0 m} \\
0
\end{array}\right]=\left[\begin{array}{c}
\Lambda_{\rho} \\
0
\end{array}\right]+\left[\begin{array}{c}
\Lambda_{\zeta} \\
0
\end{array}\right]
$$

where the auxiliary signals $\Lambda_{\rho} \triangleq\left[\Lambda_{\rho 1}, \Lambda_{\rho 2}, \ldots, \Lambda_{\rho(m-1)}\right]^{T} \in$ $\mathbb{R}^{m-1}$ and $\Lambda_{\zeta} \triangleq\left[\Lambda_{\zeta 1}, \Lambda_{\zeta 2}, \ldots, \Lambda_{\zeta(m-1)}\right]^{T} \in \mathbb{R}^{m-1}$, and the individual elements are defined as

$$
\Lambda_{\rho i} \triangleq-\sum_{j=i+1}^{m} \bar{T}_{i j}\left(k_{s j}+1\right) r_{j}, \quad \Lambda_{\zeta i} \triangleq-\sum_{j=i+1}^{m} \bar{T}_{i j} \Omega_{j k}
$$

$\forall i=1, \ldots, m-1$, where the subscript $j$ denotes the $j$ th element of the corresponding vector, and $\Omega_{j k} \in \mathbb{R}^{m}$ is defined as

$$
\Omega_{j k} \triangleq \sum_{k=1}^{p_{1}} Y_{A j k} \hat{\theta}_{A k} \quad \forall j=1, \ldots, m .
$$

It can be shown that the following inequalities can be developed [8], [31]:

$$
\left\|\Lambda_{\rho}\right\| \leq \rho_{\Lambda 1}\|z\| \quad\left\|\Lambda_{\zeta}\right\| \leq \zeta_{1}
$$

where $z(t)$ is defined in (9), and $\rho_{\Lambda 1}, \zeta_{1} \in \mathbb{R}$ are known positive bounding constants. Note that $\rho_{\Lambda 1}$ only depends on the diagonal elements $i+1$ to $m$ of $k_{s}$ due to the strictly upper triangular nature of $\bar{T}$. After using (30) and (31), the time derivative of $\Lambda_{\zeta}$ can be expressed as

$$
\dot{\Lambda}_{\zeta}=N_{B 1}+N_{B 2}
$$

where

$$
\begin{aligned}
& N_{B 1} \triangleq-\sum_{j=i+1}^{m} \bar{T}_{i j} \sum_{k=1}^{p_{1}} \dot{Y}_{A j k} \hat{\theta}_{A k} \\
& N_{B 2} \triangleq-\sum_{j=i+1}^{m} \bar{T}_{i j} \sum_{k=1}^{p_{1}} Y_{A j k} \dot{\hat{\theta}}_{A k} .
\end{aligned}
$$

After utilizing Property 1, (24), and (26), the following inequalities can be developed:

$$
\left\|N_{B 1}\right\| \leq \zeta_{2} \quad\left\|N_{B 2}\right\| \leq \zeta_{3}\|r\|
$$

where $\zeta_{2}, \zeta_{3} \in \mathbb{R}$ are known positive bounding constants.

Based on (29), the closed-loop error system can be expressed as

$$
S^{-1} \dot{r}=\tilde{N}_{2}+N_{d 2}+Y_{A} \tilde{\theta}_{A}-\left(k_{s}+I_{m \times m}\right) r-T \dot{\mu}_{1}-e
$$

where

$$
\tilde{N}_{2}=\tilde{N}_{1}+\left[\begin{array}{c}
\Lambda_{\rho} \\
0
\end{array}\right] \quad N_{d 2}=N_{d 1}+\left[\begin{array}{c}
\Lambda_{\zeta} \\
0
\end{array}\right] .
$$

Based on (19), (32), and (38), the following inequalities can be developed:

$$
\begin{aligned}
\left\|\tilde{N}_{2}\right\| & \leq \rho_{2}\|z\| \quad\left\|N_{d 2}\right\| \leq \zeta_{N_{d 2}} \\
\left\|\dot{N}_{d 2}\right\| & \leq \zeta_{\dot{N}_{d 1}}+\zeta_{2}+\zeta_{3}\|r\|
\end{aligned}
$$


where $\rho_{2}, \zeta_{N_{d 2}} \in \mathbb{R}$ are known positive bounding constants, and $\zeta_{\dot{N}_{d 1}}, \zeta_{2}$, and $\zeta_{3}$ are introduced in (19) and (36).

\section{Stability ANALYSIS}

Theorem 1: The adaptive controller given in (14), (22)-(24) ensures that the output tracking error is regulated in the sense that

$$
\|e(t)\| \rightarrow 0 \text { as } t \rightarrow \infty
$$

provided the control gain matrix $k_{s}$ introduced in (22) is selected sufficiently large (see the subsequent proof), $\alpha$ is selected to satisfy the following sufficient condition:

$$
\alpha \geq \frac{1}{2} \beta_{0}
$$

and the control gains $\beta$ and $\beta_{0}$ are selected to satisfy the following sufficient conditions:

$$
\beta>\frac{1}{\varepsilon}\left(\zeta_{N_{d 2}}+\frac{1}{\alpha} \zeta_{\dot{N}_{d 1}}+\frac{1}{\alpha} \zeta_{2}\right) \quad \beta_{0}>\zeta_{3}
$$

where $\lambda_{\min }(\cdot)$ denotes the minimum eigenvalue of the argument, $\beta$ is introduced in (23), $\beta_{0} \in \mathbb{R}$ is introduced in (45), $\rho_{2}$, $\zeta_{N_{d 2}}, \zeta_{\dot{N}_{d 1}}, \zeta_{2}$, and $\zeta_{3}$ are introduced in (19), (36), and (38), and $\varepsilon$ is introduced in (17). A detailed derivation of the gain conditions in (42) can be found in the Appendix.

Proof: Let $\mathcal{D} \subset \mathbb{R}^{2 m+p_{1}+1}$ be a domain containing $w(t)=$ 0 , where $w(t) \in \mathbb{R}^{2 m+p_{1}+1}$ is defined as

$$
w(t) \triangleq\left[z^{T}(t) \quad \tilde{\theta}_{A}^{T}(t) \quad \sqrt{P(t)}\right]^{T}
$$

where the auxiliary function $P(t) \in \mathbb{R}$ is defined as

$$
P(t) \triangleq \beta Q|e(0)|-e(0)^{T} N_{d 2}(0)-\int_{0}^{t} L(\tau) d \tau
$$

where $|\cdot|$ denotes the 1-norm of a vector, $Q$ is defined in (17), and the auxiliary function $L(t) \in \mathbb{R}$ is defined as

$$
L(t) \triangleq r^{T}\left(N_{d 2}(t)-T \dot{\mu}_{1}\right)-\beta_{0}\|e\|\|r\| .
$$

Let $V(w, t): \mathcal{D} \times[0, \infty) \rightarrow \mathbb{R}$ be a continuously differentiable, radially unbounded function defined as

$$
V \triangleq \frac{1}{2} e^{T} e+\frac{1}{2} r^{T} S^{-1} r+\frac{1}{2} \tilde{\theta}_{A}^{T} \Gamma_{A}^{-1} \tilde{\theta}_{A}+P
$$

which is positive definite provided the sufficient condition in (42) is satisfied. After taking the time derivative of (46) and utilizing (6), (37), (44), and (45), $\dot{V}(w, t)$ can be expressed as

$$
\begin{aligned}
\dot{V}(w, t)= & -\alpha e^{T} e+r^{T} \tilde{N}_{2}-r^{T}\left(k_{s}+I_{m \times m}\right) r \\
& +\beta_{0}\|e\|\|r\|-\tilde{\theta}_{A}^{T} \Gamma_{A}^{-1} \hat{\hat{\theta}}_{A}+r^{T} Y_{A} \tilde{\theta}_{A} .
\end{aligned}
$$

Based on the fact that

$$
\|e\|\|r\| \leq \frac{1}{2}\|e\|^{2}+\frac{1}{2}\|r\|^{2}
$$

$\dot{V}(w, t)$ can be upper bounded as follows:

$$
\begin{aligned}
\dot{V}(w, t) & \leq-\left(\alpha-\frac{1}{2} \beta_{0}\right)\|e\|^{2}+r^{T} \tilde{N}_{2}+\frac{1}{2} \beta_{0}\|r\|^{2} \\
- & \left(\lambda_{\min }\left(k_{s}\right)+1\right)\|r\|^{2}-\tilde{\theta}_{A}^{T} \Gamma_{A}^{-1} \dot{\hat{\theta}}_{A}+r^{T} Y_{A} \tilde{\theta}_{A} .
\end{aligned}
$$

After utilizing (24) and (39), $\dot{V}(w, t)$ can be upper bounded as

$$
\begin{aligned}
\dot{V}(w, t) \leq & -\lambda_{1}\|z\|^{2} \\
& -\left[\left(\lambda_{\min }\left(k_{s}\right)-\frac{1}{2} \beta_{0}\right)\|r\|^{2}-\rho_{2}\|r\|\|z\|\right]
\end{aligned}
$$

where $\lambda_{1} \triangleq \min \left\{\alpha-(1 / 2) \beta_{0}, 1\right\}$. Completing the squares for the bracketed terms in (50) yields

$$
\dot{V}(w, t) \leq-\left(\lambda_{1}-\frac{\rho_{2}^{2}}{4\left(\lambda_{\min }\left(k_{s}\right)-\frac{1}{2} \beta_{0}\right)}\right)\|z\|^{2} .
$$

The inequality in (51) can be used to show that $V(w, t) \in$ $\mathcal{L}_{\infty}$; hence, $e(t), r(t), \tilde{\theta}_{A}(t), P(t) \in \mathcal{L}_{\infty}$. Given that $e(t), r(t) \in \mathcal{L}_{\infty}$, standard linear analysis methods can be used to prove that $\dot{e}(t) \in \mathcal{L}_{\infty}$ from (6). Since $e(t), \dot{e}(t) \in \mathcal{L}_{\infty},(5)$ can be used along with the assumption that $y_{m}(t), \dot{y}_{m}(t) \in \mathcal{L}_{\infty}$ to prove that $y(t), \dot{y}(t) \in \mathcal{L}_{\infty}$. Since $\theta_{A} \in \mathcal{L}_{\infty}$, the assumption that $x_{m}(t), \dot{x}_{m}(t) \in \mathcal{L}_{\infty}$ can be used along with (21) to prove that $Y_{A}(t) \in \mathcal{L}_{\infty}$. Given that $r(t), \hat{\theta}_{A}(t), Y_{A}(t) \in \mathcal{L}_{\infty}$, the assumption that $(C \hat{B})^{-1} \in \mathcal{L}_{\infty}$ can be used along with the time derivative of (22) to show that $(C \hat{B})^{-1} \dot{\mu}_{0}(t) \in \mathcal{L}_{\infty}$. Since $(C \hat{B})^{-1} \dot{\mu}_{0}(t) \in \mathcal{L}_{\infty}$ and the time derivative of $(23)$ can be used to show that $\dot{\mu}_{1}(t) \in \mathcal{L}_{\infty}$, [36, Eq. 2.78] can be used to show that $(C \hat{B})^{-1}\left(\dot{\mu}_{0}(t)-\dot{\mu}_{1}(t)\right)$ can be upper bounded as $\left\|(C \hat{B})^{-1}\left(\dot{\mu}_{0}(t)-\dot{\mu}_{1}(t)\right)\right\| \leq G, \forall t \geq 0$, where $G \in \mathbb{R}^{+}$is a bounding constant. Given that $\left\|(C \hat{B})^{-1}\left(\dot{\mu}_{0}(t)-\dot{\mu}_{1}(t)\right)\right\| \leq G$, the time derivative of (14) can be used to upper bound the elements $\dot{u}_{i}(t) \forall i=1, \ldots, m$ of $\dot{u}(t)$ as $\dot{u}_{i}(t) \leq-\alpha u_{i}(t)+G$. [37, Th. 1.1] can then be utilized to prove that $u(t) \in \mathcal{L}_{\infty}$. Hence, (37) can be used to show that $\dot{r}(t) \in \mathcal{L}_{\infty}$. Since $\dot{e}(t), \dot{r}(t) \in \mathcal{L}_{\infty},(9)$ can be used to show that $z(t)$ is uniformly continuous. Since $z(t)$ is uniformly continuous, $V(w, t)$ is radially unbounded, and (46) and (51) can be used to show that $z(t) \in \mathcal{L}_{\infty} \cap \mathcal{L}_{2}$, Barbalat's Lemma [38] can be invoked to state that

$$
\|z(t)\| \rightarrow 0 \text { as } t \rightarrow \infty \quad \forall w(0) \in \mathbb{R}^{2 m+p_{1}+1} .
$$

Based on the definition of $z(t),(52)$ can be used to show that

$$
\|e(t)\| \rightarrow 0 \text { as } t \rightarrow \infty \quad \forall w(0) \in \mathbb{R}^{2 m+p_{1}+1} .
$$

\section{Simulation Results}

A numerical simulation was created, which illustrates the applicability and performance of the developed control law for an unmanned air vehicle (UAV). The simulation is based on the state-space system given in (1) and (2), where the state matrix 
$A$, input authority matrix $B$, and nonlinear disturbance function $f(x, t)$ are defined as in (1).

While a vertical wind gust can effect the aerodynamic properties of the aircraft by changing the angle of attack, the aerodynamic angle of attack was shown to fluctuate by less than 5 degrees in the presence of the wind gust tested in this simulation. Since the aerodynamic angle of attack remains sufficiently small during closed-loop controller operation, it is assumed that the nonlinearly disturbed LTI model given in (1) adequately represents the Osprey aircraft in the presence of wind gusts for the maneuvers being tested in this numerical simulation.

Since the Osprey is a straight-winged aircraft with an aspect ratio of 8.53, [39, Fig. 4.15] can be used to show that the Osprey does not have an unstable pitch break. Moreover, the aerodynamic angle of attack response of the Osprey remains small during closed-loop control. Based on these facts, Assumption 2 is mild for this aircraft in the sense that the Osprey is unlikely to lose controllability while performing the maneuvers tested in this simulation.

The reference model for the simulation is represented by the state space system given in (3)-(4), where the state matrix $A_{m}$ and input matrix $B_{m}$ are designed with the specific purpose of decoupling the velocity and pitch rate within the longitudinal system as well as decoupling the roll rate and yaw rate within the lateral system. In addition to this criterion, the design is intended to exhibit favorable transient response characteristics (e.g., low overshoot, fast rise time, and settling time, etc.) and to achieve zero steady-state error [7], [13]. Simultaneous and uncorrelated commands are input into each of the longitudinal and lateral model simulations to illustrate that each model behaves as two completely decoupled second order systems.

Based on the standard assumption that the longitudinal and lateral modes of the aircraft are decoupled, the state-space model for the Osprey can be represented using (1) and (2), where the state, input, and output matrices of the longitudinal and lateral subsystems are denoted $A_{\text {lon }}, A_{\text {lat }} \in \mathbb{R}^{4 \times 4}, B_{\text {lon }}$, $B_{\text {lat }} \in \mathbb{R}^{4 \times 2}$, and $C_{\text {lon }}, C_{\text {lat }} \in \mathbb{R}^{2 \times 4}$, respectively. The state-vector $x(t) \in \mathbb{R}^{8}$ is defined as $x \triangleq\left[\begin{array}{ll}x_{\text {lon }}^{T} & x_{\text {lat }}^{T}\end{array}\right]^{T}$, where $x_{\text {lon }}(t), x_{\text {lat }}(t) \in \mathbb{R}^{4}$ denote the longitudinal and lateral state vectors defined as $x_{\text {lon }} \triangleq\left[\begin{array}{llll}V & \alpha & q & \theta\end{array}\right]^{T}$ and $x_{\text {lat }} \triangleq\left[\begin{array}{llll}\beta & p & r & \phi\end{array}\right]^{T}$, where the components of the state are defined as in [7]. The control input vector is defined as

$$
u \triangleq\left[\begin{array}{llll}
\delta_{\text {elev }} & \delta_{\text {thrust }} & \delta_{\text {ail }} & \delta_{\text {rud }}
\end{array}\right]^{T} .
$$

In (54), $\delta_{\text {elev }}(t) \in \mathbb{R}$ denotes the elevator deflection angle, $\delta_{\text {thrust }}(t) \in \mathbb{R}$ is the control thrust, $\delta_{\text {ail }}(t) \in \mathbb{R}$ is the aileron deflection angle, and $\delta_{\text {rud }}(t) \in \mathbb{R}$ is the rudder deflection angle. The state and input matrices for the longitudinal and lateral dynamic models of the Osprey fixed-wing aircraft were experimentally determined at a cruising speed of $25 \mathrm{~m} / \mathrm{s}$ at an altitude of $60 \mathrm{~m}$ (see [7], [13] for details). The nonlinear disturbance terms, denoted $f\left(x_{\text {lon }}\right)$ and $f\left(x_{\text {lat }}\right)$, are defined as

$$
\begin{aligned}
f\left(x_{\text {lon }}\right) & =\left[\begin{array}{llll}
-9.81 \sin \theta & 0 & 0 & 0
\end{array}\right]^{T}+g(x)^{T} \\
f\left(x_{\text {lat }}\right) & =\left[\begin{array}{llll}
0.39 \sin \phi & 0 & 0 & 0
\end{array}\right]^{T}
\end{aligned}
$$

where $g(x) \in \mathbb{R}^{4}$ represents a disturbance due to a discrete vertical wind gust as defined in [40], and the trigonometric terms in $f\left(x_{\text {lon }}\right)$ and $f\left(x_{\text {lat }}\right)$ represent nonlinear dependence on gravity. All states, control inputs, and adaptive estimates were initialized to zero for the simulation.

The feedforward estimates $\hat{B}_{\text {lon }}$ and $\hat{B}_{\text {lat }}$ were selected as

$$
\hat{B}_{\text {lon }}=\left[\begin{array}{cc}
0 & 0.2 \\
0.1 & 0 \\
-1.5 & 0 \\
0 & 0
\end{array}\right] \quad \hat{B}_{\text {lat }}=\left[\begin{array}{cc}
0 & 0 \\
1 & 0 \\
-0.5 & -0.25 \\
0 & 0
\end{array}\right] \text {. }
$$

Remark 1: For the choices for $\hat{B}_{\text {lon }}$ and $\hat{B}_{\text {lat }}$ given in (57), Assumption 4 is satisfied. Specifically, the choice for $\hat{B}_{\text {lon }}$ yields the following:

$$
\min _{j \in(1, m-1)}\left\{\left|T_{j j}\right|-\sum_{k=j+1}^{m}\left|T_{j k}\right|\right\}=0.997>\varepsilon>0
$$

and the choice for $\hat{B}_{\text {lat }}$ yields

$$
\min _{j \in(1, m-1)}\left\{\left|T_{j j}\right|-\sum_{k=j+1}^{m}\left|T_{j k}\right|\right\}=0.890>\varepsilon>0 .
$$

In order to develop a realistic stepping stone to an actual experimental demonstration of the proposed controller, the simulation parameters were selected based on detailed data analyses and specifications. The sensor noise values are based on Cloud Cap Technology's Piccolo Autopilot and analysis of data logged during straight and level flight. These values are also corroborated with the specifications given for Cloud Cap Technology's Crista Inertial Measurement Unit (IMU). The objectives for the longitudinal controller simulation are to track pitch rate and forward velocity commands. Fig. 1 shows the reference and actual tracking results during closed-loop operation of the longitudinal and lateral controllers. Fig. 2 shows the control effort used during closed-loop operation of the longitudinal and lateral controllers.

\section{CONCLUSION}

A controller is presented, which achieves global asymptotic tracking of a model reference system, where the plant dynamics contain an uncertain input matrix and an unknown additive disturbance. This result represents application of a continuous control strategy in a robust ADI framework to a dynamic system with nonlinear, non-vanishing, nonlinearly parameterizable disturbances, where the control input is multiplied by a non-square, column deficient matrix containing parametric uncertainty. By exploiting partial knowledge of the dynamic model, we are able to prove a global asymptotic tracking result while weakening some common restrictive assumptions concerning the system uncertainty. A Lyapunov-based stability analysis is provided to verify the theoretical result, and numerical simulation results are provided to demonstrate the performance of the proposed controller. Future efforts will focus on relaxing the limiting restrictions on the nonlinear disturbance. In addition, future work will focus on control design for systems in the form of (1), where 

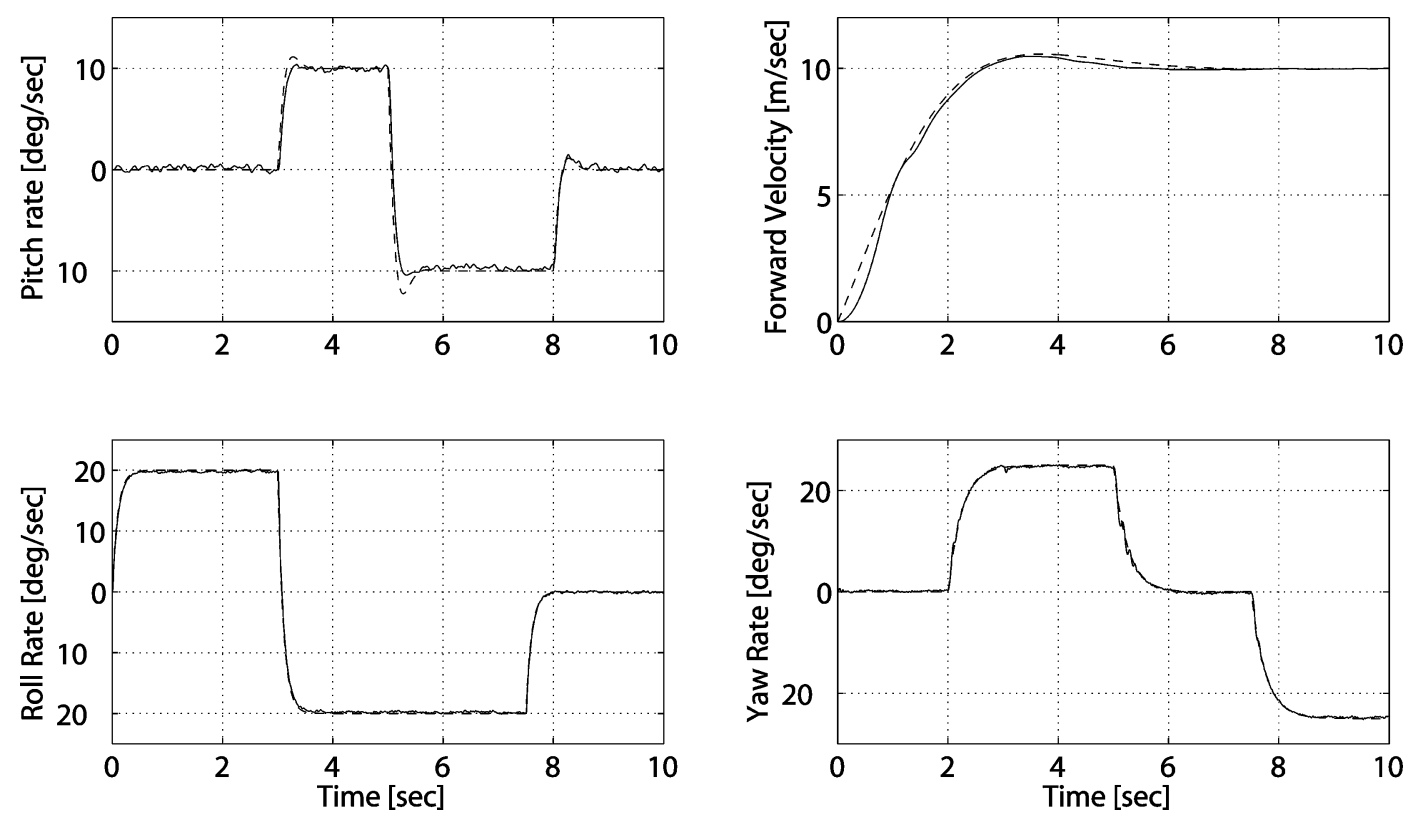

Fig. 1. Reference model (dashed line) and actual (solid line) output pitch rate (top left) and forward velocity (top right) responses during closed-loop longitudinal controller operation, and roll rate (bottom left) and yaw rate (bottom right) responses achieved during closed-loop lateral controller operation.
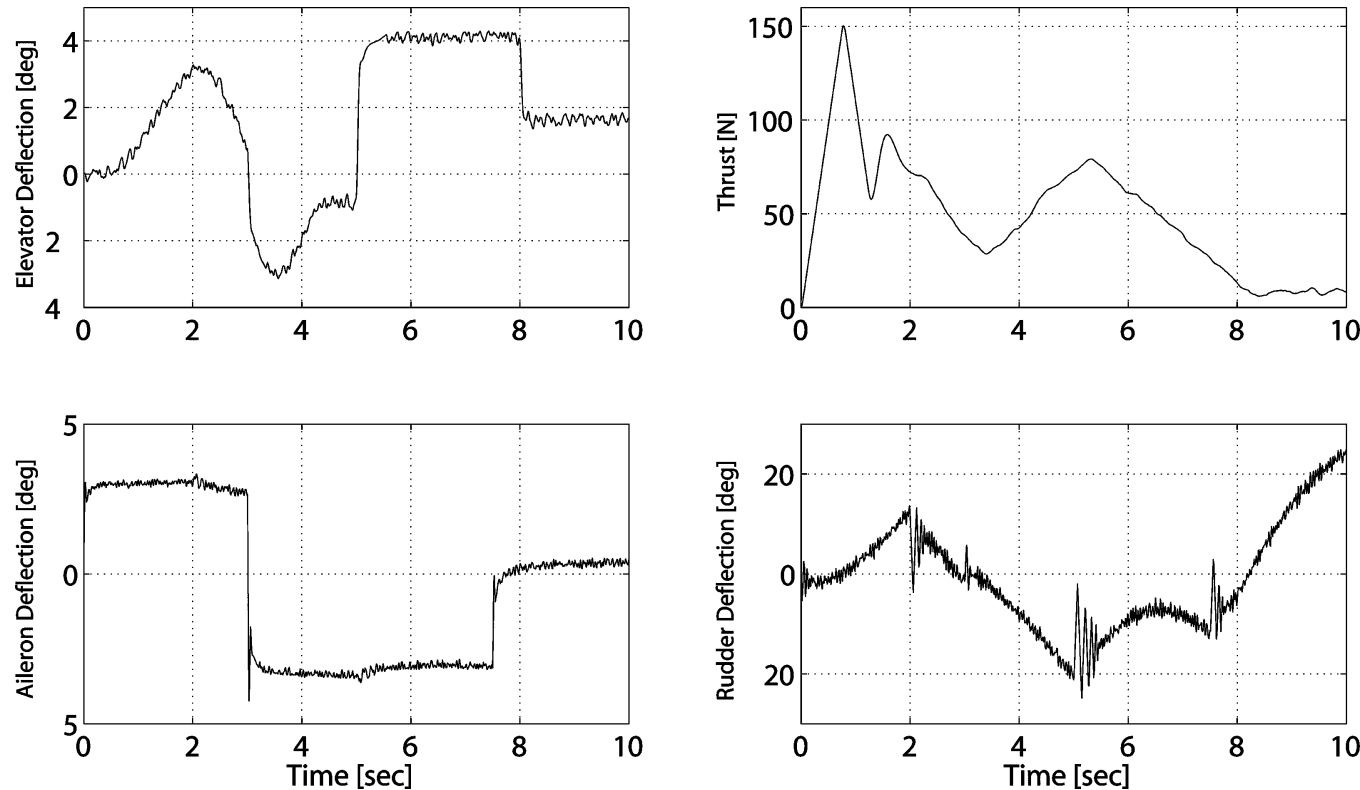

Fig. 2. Control input elevator deflection angle $\delta_{\text {elev }}(t)$ (top left) and thrust $\delta_{\text {thrust }}(t)$ (top right) used during closed-loop longitudinal controller operation, and aileron $\delta_{\text {ail }}(t)$ (bottom left) and rudder $\delta_{\text {rud }}(t)$ (bottom right) deflection angle used during closed-loop lateral controller operation.

there are fewer inputs than outputs (i.e., where $u(t) \in \mathbb{R}^{p}$ and $C \in \mathbb{R}^{m \times n}$ with $p<m$ ).

\section{APPENDIX}

Lemma 1: Provided the control gains $\beta$ and $\beta_{0}$ introduced in (23) and (45), respectively, are selected according to the sufficient conditions in (42), the following inequality can be obtained:

$$
\int_{0}^{t} L(\tau) d \tau \leq \beta Q|e(0)|-e(0)^{T} N_{d 2}(0) .
$$

Hence, (60) can be used to conclude that $P(t) \geq 0$, where $P(t)$ is defined in (44).
Proof: Integrating both sides of (45) yields

$$
\begin{aligned}
\int_{0}^{t} L(\tau) d \tau= & \int_{0}^{t} \sum_{i=1}^{m} r_{i}(\tau)\left(N_{d 2_{i}}(\tau)-\sum_{j=i}^{m} T_{i j} \dot{\mu}_{1_{j}}(\tau)\right) d \tau \\
& -\int_{0}^{t} \beta_{0}\|e(\tau)\|\|r(\tau)\| d \tau
\end{aligned}
$$

where $e_{i}(t), r_{i}(t), N_{d 2_{i}}(t), \dot{\mu}_{1_{i}}(t) \in \mathbb{R}$ denote the $i$ th elements of $e(t), r(t), N_{d 2}(t)$, and $\dot{\mu}_{1}(t)$, respectively, and $T_{i j}$ is introduced in (17). After substituting (6) into (61), utilizing 
(19), (36), (39), and Assumption 4, (61) can be upper bounded as

$$
\begin{aligned}
\int_{0}^{t} L(\tau) d \tau \leq & \int_{0}^{t} \sum_{i=1}^{m} \alpha\left|e_{i}(\tau)\right|\left(\zeta_{N_{d 2}}+\frac{1}{\alpha} \zeta_{\dot{N}_{d 1}}+\frac{1}{\alpha} \zeta_{2}-\varepsilon \beta\right) d \tau \\
& +\int_{0}^{t} \zeta_{3}\|e(\tau)\|\|r(\tau)\| d \tau \\
& +\sum_{i=1}^{m}\left|e_{i}(t)\right|\left(\zeta_{N_{d 2}}-\varepsilon \beta\right) \\
& +\sum_{i=1}^{m}\left(\beta Q\left|e_{i}(0)\right|-e_{i}(0) N_{d 2_{i}}(0)\right) \\
& -\int_{0}^{t} \beta_{0}\|e(\tau)\|\|r(\tau)\| d \tau
\end{aligned}
$$

where the facts that $\left(\partial N_{d 2}(\tau) / \partial \tau\right)=\left(\partial N_{d 1}(\tau) /\right.$ $\partial \tau)+N_{B 1}(\tau)+N_{B 2}(\tau)$ and $\sum_{j=i}^{m} T_{i j} \dot{\mu}_{1_{j}}(t)=$ $\beta\left(\operatorname{sgn}\left(e_{i}(t)\right)+\sum_{j=i+1}^{m} \bar{T}_{i j} \operatorname{sgn}\left(e_{j}(t)\right)\right) \forall i=1, \ldots, m-1$, were utilized, and $\varepsilon$ and $Q$ are defined as in (17). Thus, it is clear from (62) that if $\beta$ and $\beta_{0}$ satisfy (42), then (60) holds.

\section{REFERENCES}

[1] Z. Szabo, P. Gaspar, and J. Bokor, "Tracking design for Wiener systems based on dynamic inversion," in Proc. Int. Conf. Control Appl., Munich, Germany, Oct. 2006, pp. 1386-1391.

[2] A. D. Ngo and D. B. Doman, "Dynamic inversion-based adaptive/reconfigurable control of the X-33 on ascent," in Proc. IEEE Aerosp. Conf., Big Sky, MT, Mar. 2006, pp. 2683-2697.

[3] M. D. Tandale and J. Valasek, "Adaptive dynamic inversion control of a linear scalar plant with constrained control inputs," in Proc. Amer. Control Conf., Portland, OR, Jun. 2005, pp. 2064-2069.

[4] E. Lavretsky and N. Hovakimyan, "Adaptive dynamic inversion for nonaffine-in-control systems via time-scale separation: Part II," in Proc. Amer. Control Conf., Portland, OR, Jun. 2005, pp. 3548-3553.

[5] J. Cheng, H. Li, and Y. Zhang, "Robust low-cost sliding mode overload control for uncertain agile missile model," in Proc. World Congr. Intell. Control Autom., Dalian, China, Jun. 2006, pp. 2185-2188.

[6] Z. Liu, F. Zhou, and J. Zhou, "Flight control of unpowered flying vehicle based on robust dynamic inversion," in Proc. Chinese Control Conf., Heilongjiang, China, Aug. 2006, pp. 693-698.

[7] W. MacKunis, M. K. Kaiser, P. M. Patre, and W. E. Dixon, "Asymptotic tracking for aircraft via an uncertain dynamic inversion method," in Proc. Amer. Control Conf., Seattle, WA, Jun. 2008, pp. 3482-3487.

[8] X. Zhang, D. Dawson, M. de Queiroz, and B. Xian, "Adaptive control for a class of MIMO nonlinear systems with non-symmetric input matrix," in Proc. Int. Conf. Control Appl., Taipei, Taiwan, Sep. 2004, pp. $1324-1329$.

[9] A. S. Morse, "A gain matrix decomposition and some of its properties," Syst. Control Lett., vol. 21, no. 1, pp. 1-10, Jul. 1993.

[10] R. R. Costa, L. Hsu, A. K. Imai, and P. Kokotovic, "Lyapunov-based adaptive control of MIMO systems," Automatica, vol. 39, no. 7, pp. 1251-1257, Jul. 2003

[11] A. Behal, V. M. Rao, and P. Marzocca, "Adaptive control for a nonlinear wing section with multiple flaps," J. Guid., Control, Dyn., vol. 29, no. 3, pp. 744-748, 2006.

[12] C. C. Cheah, C. Liu, and J. J. E. Slotine, "Adaptive Jacobian tracking control of robots with uncertainties in kinematic, dynamic and actuator models," IEEE Trans. Autom. Control, vol. 51, no. 6, pp. 1024-1029, Jun. 2006.

[13] W. MacKunis, M. K. Kaiser, P. M. Patre, and W. E. Dixon, "Adaptive dynamic inversion for asymptotic tracking of an aircraft reference model," presented at the AIAA Guid., Nav., Control Conf., Honolulu, HI, 2008, AIAA-2008-6792.

[14] K. K. Reddy, J. Chen, A. Behal, and P. Marzocca, "Multi-input/multioutput adaptive output feedback control design for aeroelastic vibration suppression," J. Guid., Control, Dyn., vol. 30, no. 4, pp. 1040-1048, 2007.
[15] W. Xing and S. N. Singh, "Adaptive output feedback control of a nonlinear aeroelastic structure," J. Guid., Control, Dyn., vol. 23, no. 6, pp. 1109-1116, Nov.-Dec. 2000.

[16] K. W. Lee and S. N. Singh, "Global robust control of an aeroelastic system using output feedback," J. Guid., Control, Dyn., vol. 30, no. 1 , pp. 271-275, Jan.-Feb. 2007.

[17] R. Zhang and S. N. Singh, "Adaptive output feedback control of an aeroelastic system with unstructured uncertainties," J. Guid., Control, Dyn., vol. 24, no. 3, pp. 502-509, May-Jun. 2001.

[18] S. N. Singh and M. Brenner, "Modular adaptive control of a nonlinear aeroelastic system," J. Guid., Control, Dyn., vol. 26, no. 3, pp. 443-451, May-Jun. 2003.

[19] A. Behal, P. Marzocca, V. M. Rao, and A. Gnann, "Nonlinear adaptive control of an aeroelastic two-dimensional lifting surface," J. Guid. Control, Dyn., vol. 29, no. 2, pp. 382-390, Mar.-Apr. 2006.

[20] D.-G. Choe and J.-H. Kim, "Pitch autopilot design using model-following adaptive sliding mode control," J. Guid., Control, Dyn., vol. 25, no. 4, pp. 826-829, Mar. 2002.

[21] N. Kim, A. J. Calise, N. Hovakimyan, and J. V. R. Prasad, "Adaptive output feedback for high-bandwidth flight control," J. Guid., Control, Dyn., vol. 25, no. 6, pp. 993-1002, Nov.-Dec. 2002.

[22] N. Hovakimyan, E. Lavretsky, A. J. Calise, and R. Sattigeri, "Decentralized adaptive output feedback control via input/output inversion," in Proc. IEEE Conf. Dec. Control, Maui, HI, Dec. 2003, pp. 1699-1704.

[23] R. Rysdyk, F. Nardi, and A. J. Calise, "Robust adaptive flight control applications using neural networks," in Proc. Amer. Control Conf., San Diego, CA, Jun. 1999, pp. 2595-2599.

[24] S. S. Ge, G. Y. Li, and N. Xi, "Direct adaptive control for a class of multi-input and multi-output nonlinear systems using neural networks," in Proc. IEEE Conf. Dec. Control, Maui, HI, Dec. 2003, pp. 2716-2721.

[25] J. Phuah, X. Lu, and T. Yahagi, "Model reference adaptive control for multi-input multi-output nonlinear systems using neural networks," in Proc. IEEE/ASME Int. Conf. Adv. Intell. Mechatron., Port Island, Kobe, Japan, Jul. 2003, pp. 12-16.

[26] L. Sonneveldt, E. R. van Oort, Q. P. Chu, and J. A. Mulder, "Nonlinear adaptive trajectory control applied to an F-16 model," J. Guid., Control, Dyn., vol. 32, no. 1, pp. 25-39, Jan.-Feb. 2009.

[27] R. Nelson, Flight Stability and Automatic Control, 2nd ed. New York: McGraw-Hill, 1998.

[28] F. L. Lewis, C. T. Abdallah, and D. M. Dawson, Control of Robot Manipulators. New York: MacMillan, 1993.

[29] P. M. Patre, W. MacKunis, C. Makkar, and W. E. Dixon, "Asymptotic tracking for systems with structured and unstructured uncertainties," IEEE Trans. Control Syst. Technol., vol. 16, no. 2, pp. 373-379, Mar. 2008.

[30] B. Xian, D. M. Dawson, M. S. de Queiroz, and J. Chen, "A continuous asymptotic tracking control strategy for uncertain nonlinear systems," IEEE Trans. Autom. Control, vol. 49, no. 7, pp. 1206-1211, Jul. 2004.

[31] X. Zhang, A. Behal, D. M. Dawson, and B. Xian, "Output feedback control for a class of uncertain MIMO nonlinear systems with nonsymmetric input gain matrix," in Proc. Conf. Dec. Control Eur. Control Conf., Seville, Spain, Dec. 2005, pp. 7762-7767.

[32] M. Bridges, D. M. Dawson, and C. Abdallah, "Control of rigid-link flexible-joint robots: A survey of backstepping approaches," J. Robot. Syst., vol. 12, pp. 199-216, 1995.

[33] R. Lozano and B. Brogliato, "Adaptive control of robot manipulators with flexible joints," IEEE Trans. Autom. Control, vol. 37, no. 2, pp. 174-181, Feb. 1992.

[34] W. Dixon, "Adaptive regulation of amplitude limited robot manipulators with uncertain kinematics and dynamics," IEEE Trans. Autom. Control, vol. 52, no. 3, pp. 488-493, Mar. 2007.

[35] E. Zergeroglu, W. Dixon, A. Behal, and D. Dawson, "Adaptive setpoint control of robotic manipulators with amplitude-limited control inputs," Robotica, vol. 18, pp. 171-181, 2000.

[36] G. Tao, Adaptive Control Design and Analysis, S. Haykin, Ed. New York: Wiley-Interscience, 2003.

[37] D. Dawson, M. Bridges, and Z. Qu, Nonlinear Control of Robotic Systems for Environmental Waste and Restoration. Englewood Cliffs, NJ: Prentice-Hall, 1995.

[38] H. K. Khalil, Nonlinear Systems, 3rd ed. Upper Saddle River, NJ: Prentice-Hall, 2002.

[39] J. Roskam and C.-T. E. Lan, Airplane Aerodynamics and Performance, C. E. Lan, Ed., 3rd ed. Lawrence, KS: DARcorporation, 1997.

[40] "Airworthiness standards: Transport category airplanes," in Federal Aviation Regulations-Part 25. Washington, DC: Department of Transportation, 1996 